\title{
OUTCOME OF TREATMENT WITH CONCURRENT WHOLE BRAIN RADIOTHERAPY AND TEMOZOLOMIDE IN BRAIN METASTASIS
}

\author{
$M Y^{1}$, Islam $\mathrm{MA}^{2}$, Rokonuzzaman $\mathrm{SM}^{3}$, Rahman $\mathrm{MH}^{4}$, Rahman $\mathrm{MM}^{5}$, Chowdhury $\mathrm{RU}^{6}$, Hasan $\mathrm{AA}^{7}$
}

\begin{abstract}
Introduction: Brain metastasis represents the most common form of intracranial tumor and causes significant morbidity and mortality in cancer patients. This prospective study was carried out at Radiation Oncology Department, Combined Military Hospital Dhaka from January 2010 to December 2012. The concurrent chemotherapy and radiotherapy have shown better outcome and improved the quality of life.
\end{abstract}

Objectives: To evaluate the efficacy and toxicity of concurrent treatment with whole brain radiotherapy (WBRT) and temozolomide (TMZ) in patients with brain metastasis.

Methods: Sixty patients with multiple brain metastases were enrolled and received WBRT with 30 Gray (Gy) in ten fractions with concurrent TMZ ( $75 \mathrm{mg} / \mathrm{m} 2 /$ day) for ten days.

Results: Remarkable symptomatic relief occurred in eighteen (72\%) patients of headache, nine $(60 \%)$ patients of altered mental status, thirteen $(76.5 \%)$ of vomiting, ten $(71.4 \%)$ of seizure, eleven $(68.7 \%)$ of altered sensation, seven (58.3\%) of focal weakness and two $(40 \%)$ of visual change. In relation to objective response four $(6.7 \%)$ patients had complete response, twenty three (38.3\%) patients had partial response while twenty one $(35 \%)$ had stable disease and twelve (20\%) had progressive disease. The overall response rate was $45 \%$. The most frequent toxicities included anorexia in twenty one (35\%), nausea in eighteen $(30 \%)$, vomiting in ten $(16.6 \%)$, lethargy in seventeen $(28 \%)$, anemia in eight $(13.3 \%)$ and neutropenia in thirteen $(21.6 \%)$ cases.
Conclusion: The concurrent treatment with whole brain radiotherapy (WBRT) and temozolomide (TMZ) in patients with brain metastasis is well tolerated with an encouraging response.

Key-words: Concurrent, whole brain radiotherapy, temozolomide, brain metastasis.

\section{Introduction}

Brain metastasis is the most common intracranial tumor in adults. They affect $10-30 \%$ of all cancer patients and represent one of the most frequent neurological complications of cancer as a major cause of morbidity and mortality ${ }^{1,2}$. The probability of brain metastasis depends on the type of primary cancer. Lung cancer, malignant melanoma, renal cell carcinoma, breast carcinoma, and colorectal carcinoma are the most common tumors associated with brain metastasis ${ }^{3}$. Many of the patients with lung cancer land with brain metastasis. Most of the brain metastases are detected after the primary tumor has been diagnosed and less frequently brain metastasis appears as the first symptom. Therapeutic options depend on state of the primary tumor, extent of metastasis and performance of the patients. Frequently symptomatic care to relieve neurologic symptoms includes the use of corticosteroids and anticonvulsants ${ }^{4}$. Single brain lesion may be treated by surgery or radiosurgery, in addition to management of the primary tumor. The presence of multiple metastases often preludes the option of surgical resection. In contrast, radiation therapy has been shown to be efficacious in treating brain metastasis regardless of the primary tumor histology, including metastases derived from tumors

1. Lt Col Md. Yousuf Ali, MBBS, FCPS, Classified Specialist in Radiotherapy, Dept of Radiation Oncology, CMH, Dhaka; 2. Lt Col Md. Aminul Islam, MBBS, MS, Classified Specialist in Surgery, CMH, Dhaka; 3. Maj S. M. Rokonuzzaman, MBBS, FCPS, Classified Specialist in Radiation Oncology,, Dept of Radiation Oncology, CMH, Dhaka; 4. Brig Gen Md. Habibur Rahman, MBBS, FCPS, Prof and Adviser Spl in Surgery, AFMC, Dhaka; 5. Col Md. Mahbubur Rahman, MBBS, FCPS, Prof of Surgery, AFMC, Dhaka; 6. Lt Col Rukun Uddin Chowdhuyry, MBBS, FCPS, Classified Specialist in Surgery, CMH, Dhaka; 7. Capt Arif Al-Hasan, MBBS, OJT (Radiation Oncology), AFMI, Dhaka. 
considered to be radioresistant ${ }^{5}$. The ability of radiation to effectively treat brain metastasis of any tumor histology is unique among currently available therapies, and thus represents an important palliative option for patients with brain metastasis by alleviating symptoms, decreasing the use of corticosteroids needed to control tumor-associated oedema, and potentially improving overall survival ${ }^{6,7}$. For these reasons, radiation therapy has become the main modality in the treatment of metastatic brain lesions. The limited ability of most chemotherapy drugs to cross the blood-brain barrier is one of the principal reasons of less effectiveness of these agents in brain than in extra-cranial sites ${ }^{8}$. TMZ has excellent central nervous system penetration, and reaches the brain in therapeutic concentrations ${ }^{9}$ and suggested for a potential application in combination with radiotherapy as treatment for brain metastases. Studies combining TMZ with whole-brain radiotherapy (WBRT) reported more favorable response rates ranging from 0.176 to 0.959 with median overall survival ranging from 4.1 to 12 months. In these trials, temozolomide might be shown to possess a radiosensitizing effect ${ }^{10,11}$. The concurrent use of TMZ and WBRT is well tolerated and higher response rate is achieved in patients receiving TMZ and radiotherapy versus radiotherapy alone ${ }^{12,13}$ confirming the combination of TMZ with radiation ${ }^{14}$. The primary aim of this study was to assess the efficacy and safety of the combination of TMZ and WBRT in patients with brain metastasis at our hospital setup.

\section{Materials and Methods}

This prospective study was carried out at Radiation. Oncology Department, Combined Military Hospital, Dhaka from January 2010 to December 2012. The study population consisted of 60 cancer patients with brain metastasis. Data were collected from the patients in predesigned informed sheets and also from their documents. Patient eligibility criteria: adult patients with histologically proven primary cancer with measurable multiple brain metastasis assessable by contrast-enhanced computed tomography scan or gadolinium enhanced magnetic resonance imaging (MRI) that were not suitable for surgery or radiosurgery, were eligible for the study.
Other eligibility criteria included age $\geq 18$ years; Karnofsky performance status (KPS) $\geq 50$. Eligible patients were required to be fully recovered from previous therapy.

\section{Treatment schedule}

Planned conventional WBRT was administrated with a daily dose of $3 \mathrm{~Gy} \times 5$ days each week for two weeks to a total dose of 30 Gy (Fig-I). TMZ was administrated orally at a dosage of 75 $\mathrm{mg} / \mathrm{m}^{2} /$ day during the radiation. Treatment was continued until unacceptable toxicity. All patients received corticosteroids at the dose necessary to maintain neurologic stability, and anti-convulsants were given when indicated.

\section{Patient evaluation}

Primary end point of the study was symptom relief and secondary endpoints were objective responses six weeks after the end of concurrent TMZ and WBRT. Baseline assessments were performed before the initiation of radiation treatment. All patients underwent weekly physical and neurologic examinations during concurrent treatment and a complete clinical evaluation, laboratory tests, KPS and CT or MRI 45 days after WBRT. Objective response was evaluated according to the WHO criteria ${ }^{15}$.

\section{Results}

Among 60 patients incidence was more in older patients. Number of male patients were more than female (Table-I).

Table-I: Patient's characteristics $(n=60)$.

\begin{tabular}{|l|l|}
\hline Age (yrs) & Number of Patient (\%) \\
\hline$<40$ & $14(23.3 \%)$ \\
\hline $40-60$ & $27(45 \%)$ \\
\hline$>60$ & $19(31.7)$ \\
\hline Sex & Number of Patient (\%) \\
\hline Male & $37(61.7 \%)$ \\
\hline Female & $23(38.3 \%)$ \\
\hline
\end{tabular}

Thirty four patients (56.6\%) had lung cancer, 15 $(25 \%)$ had breast cancer, $11(18.3 \%)$ had other cancers (colo-rectal, thyroid, kidney and unknown primary) (Table-II). Thirty eight (63.3\%) of 60 patients had metastases in other organs. The majority of the patients, 41 out of 60 , had received chemotherapy for primary cancer before entering the study. 
Table-II: Distribution of primary cancer that lead to BM $(n=60)$.

\begin{tabular}{|l|l|}
\hline Primary sites & Number of Patient (\%) \\
\hline Lung cancer & $34(56.6 \%)$ \\
\hline Breast cancer & $15(25 \%)$ \\
\hline other cancer & $11(18.3 \%)$ \\
\hline
\end{tabular}

The prominant symptoms were as per Table-III.

Table-III: Symptoms before WBRT and TMZ $(\mathrm{n}=60)$.

\begin{tabular}{|l|l|}
\hline Symptoms & Number of Patient (\%) \\
\hline Headache & $25(41.6 \%)$ \\
\hline Altered Mental Status & $15(25 \%)$ \\
\hline Vomiting & $17(28.3 \%)$ \\
\hline Seizure & $14(23.3 \%)$ \\
\hline Altered Sensation & $16(26.6 \%)$ \\
\hline Focal Weakness & $12(20 \%)$ \\
\hline Visual Change & $05(8.3 \%)$ \\
\hline
\end{tabular}

Most of the patients got symptomatic relief after treament (Table-IV).

Table-IV: Symptom relief after WBRT and TMZ $(n=60)$.

\begin{tabular}{|l|l|}
\hline Symptoms & Number of Patient (\%) \\
\hline Headache $(\mathrm{n}=25)$ & $18(72 \%)$ \\
\hline Altered Mental Status $(\mathrm{n}=15)$ & $9(60 \%)$ \\
\hline Vomiting $(\mathrm{n}=17)$ & $13(76.5)$ \\
\hline Seizure $(\mathrm{n}=14)$ & $10(71.4 \%)$ \\
\hline Altered Sensation $(\mathrm{n}=16)$ & $11(68.7 \%)$ \\
\hline Focal Weakness $(\mathrm{n}=12)$ & $7(58.3 \%)$ \\
\hline Visual Change $(\mathrm{n}=5)$ & $2(40 \%)$ \\
\hline
\end{tabular}

Objective response after treatment were encouraging (Table-V \& Fig-2).

Table-V: Objective response after WBRT and TMZ $(n=60)$.

\begin{tabular}{|l|l|}
\hline Response & Number of Patient (\%) \\
\hline Complete response (CR) & $04(6.7 \%)$ \\
\hline Partial response (PR) & $23(38.3 \%)$ \\
\hline Overall response (CR+PR) & $27(45 \%)$ \\
\hline Stable disease & $21(35 \%)$ \\
\hline Progressive disease & $12(20 \%)$ \\
\hline
\end{tabular}

The concurrent TMZ and WBRT were well tolerated in almost all patients. Most side effects were of grade 2 and well controlled by supportive care (Table-VI).

Table-VI: Treatment related toxicities $(n=60)$.

\begin{tabular}{|l|l|}
\hline Response & Number of Patient (\%) \\
\hline Anorexia & $2(3.3 \%)$ \\
\hline Nausea & $18(30 \%)$ \\
\hline Vomiting & $10(16.6 \%)$ \\
\hline Lethargy & $17(28.3 \%)$ \\
\hline Anemia & $8(13.3 \%)$ \\
\hline Neuropenia & $13(21.6 \%)$ \\
\hline
\end{tabular}

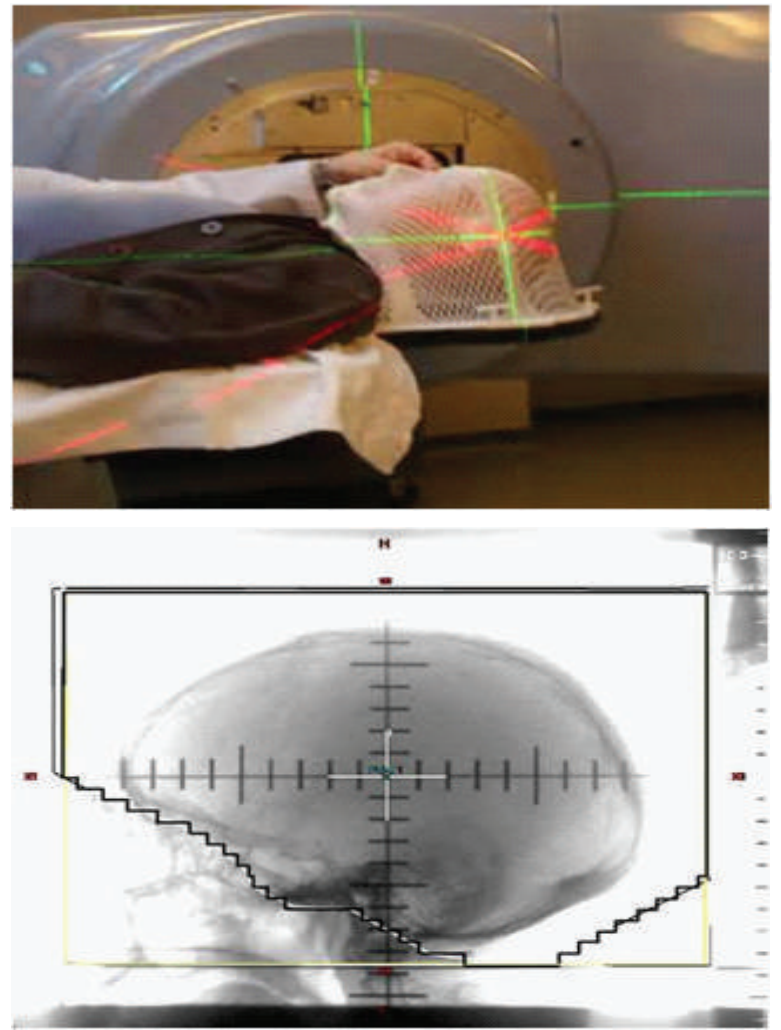

Fig-1: Radiotherapy of whole brain.
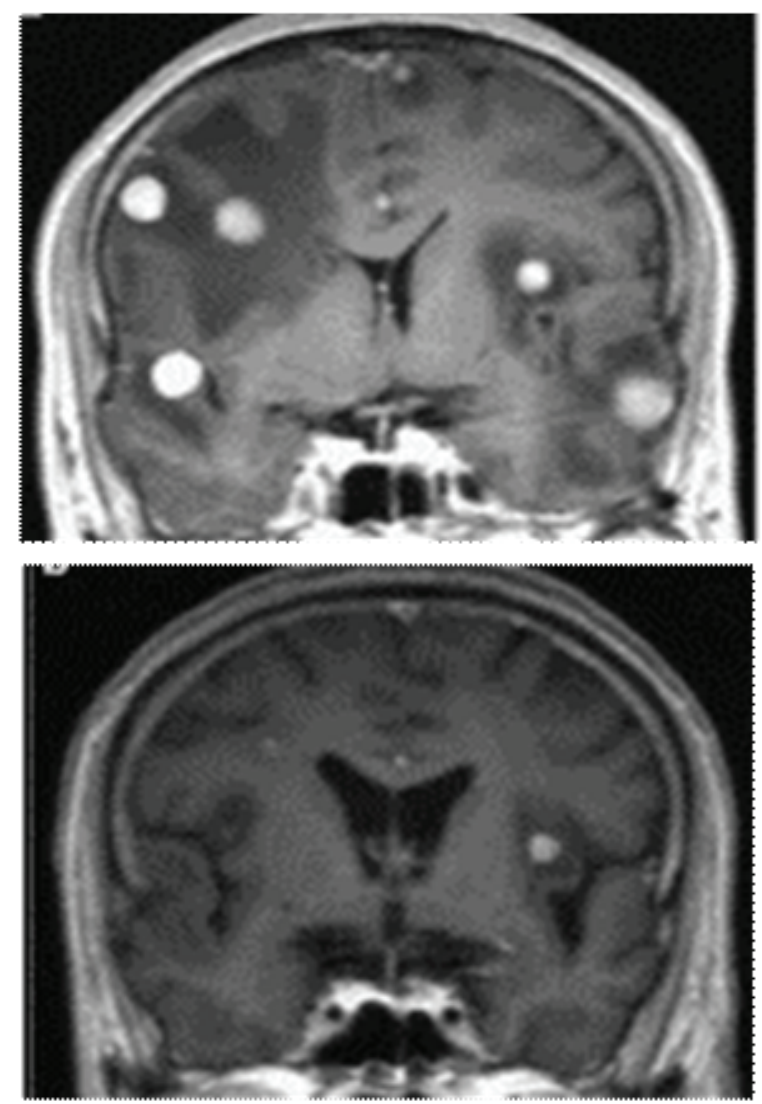

Fig-2: MRI of brain metastasis -before (up) \& $1 \frac{1}{2}$ months after treatment (down). 


\section{Discussion}

In patients with multiple cerebral metastases, WBRT is generally the treatment of choice, as it addresses both macroscopic and microscopic disease. Studies have shown an improvement in symptoms in $64-83 \%$ of patients after treatment with WBRT alone ${ }^{16,17,18}$ and have also demonstrated an increase in median overall survival from 1 month with no treatment to 3-7 months following $\mathrm{WBRT}^{7}$. WBRT has long been the mainstay of definitive treatment and patients with brain metastasis achieve relief of neurologic symptoms for a time with WBRT ${ }^{19}$. The results may be divergent due to variability in several factors including tumor histology, presenting stage, previous use of chemotherapy etc ${ }^{20}$. The efficacy and safety of TMZ concurrently with WBRT for patients with newly diagnosed brain metastasis were evaluated in recent trials. The most promising study regarding the use of TMZ with WBRT was documented in a phase II trial by Gamboa-Vignolle et al., which found WBRT and TMZ increased the objective response to $78.6 \%$ from $48.1 \%$ in patients with cerebral metastases who received WBRT alone. Additionally, the median progression free survival was found to be 11.8 months in the TMZ and WBRT arm, versus 5.6 months in the WBRT alone arm ${ }^{21}$. Our data, revealed an encouraging subjective and objective response. The overall response rate $(\mathrm{CR}+\mathrm{PR})$ was $45 \%$ with $6.7 \% \mathrm{CR}$ rate and $38.3 \% \mathrm{PR}$ rate. The results of this study suggest that the combination of WBRT and TMZ has a significant clinical activity in patients with brain metastasis. This regimen was generally well tolerated by almost all patients, including elderly patients. Fatigue is one of the most prominent acute toxicities associated with WBRT, experienced within the first days to weeks of treatment ${ }^{22}$. Other acute effects include radiation-induced alopecia and dermatitis, nausea and vomiting, and decreased appetite. Aside from radiation-induced alopecia, these acute effects are generally self-limited and resolve spontaneously or with medical management ${ }^{22}$. Cerebral Oedema is another relatively common acute adverse effect of WBRT, but is usually responsive to treatment with corticosteroids $^{23}$. Our data also revealed limited toxicity of this regimen. The most frequent toxicities included anorexia in twenty one (35\%), nausea in eighteen (30\%), vomiting in ten (16.6\%), lethargy in seventeen (28\%), anemia in eight
(13.3\%) and neutropenia in thirteen (21.6\%) cases, which resolved with supportive medications. This study suggests that the combination of WBRT and TMZ is safe and well tolerated in patients with brain metastasis.

\section{Conclusion}

The present study supports the feasibility, efficacy and safety of concurrent use of WBRT and TMZ in the treatment of patients with brain metastasis at our hospital setup. The outcome observed in this study was found comparable with similar studies abroad. The presented data looks promising and this needs to be further validated within the settings of a randomized trial. The use of WBRT in brain metastasis appears unlikely to be replaced by other therapies in the near future, and thus the clinical trials that are currently attempting to improve the efficacy and toxicity profiles of WBRT will have the potential for strong clinical application.

\section{References}

1. Patchell RA. The management of brain metastases. Cancer Treat Rev 2003; 29:533-40.

2. Zimm S, Wampler GL, Stablein D, Hazra T, Young HF. Intra-cerebral metastases in solid tumor patients. Natural history and results of treatment. Cancer 1981; 48:384-94.

3. Barnholtz-Sloan JS, Sloan AE, Davis FG, Vigneau FD, Lai $P$, Sawaya RE. Incidence proportions of brain metastases in patients diagnosed (1973 to 2001) in the Metropolitan Detroit cancer surveillance system. J Clin Oncol 2004; 22:2865-72.

4. Cappuzzo F, Mazzoni F, Maestri, Di Stefano A, Calandri C, Crino L. Medical treatment of brain metastases from solid tumours. Forum (Genova) $2000 ; 10: 137-49$.

5.Chao JH, Phillips R, Nickson JJ. Roentgen-ray therapy of cerebral metastases. Cancer 1954; 7:682-9. 
6. Bezjak A, Adam J, Panzarella T. Radiotherapy for brain metastases: Defining palliative response. Radiother Oncol 2001; 61:71-6.

7. Gaspar L, Scott C, Rotman M. Recursive partitioning analysis (RPA) of prognostic factors in three Radiation Therapy Oncology Group (RTOG) brain metastases trials. Int $\mathrm{J}$ Radiat Oncol Biol Phys 1997; 37:745-51.

8. Langer CJ, Mehta MP. Current management of brain metastases, with a focus on systemic options. J Clin Oncol 2005; 23(25):6207-19.

9. Plowman J, Waud WR, Koutsoukos AD, Rubinstein LV, Moore TD, Grever MR. Preclinical antitumor activity of temozolomide in mice: efficacy against human brain tumor xenografts and synergism with 1,3-bis (2-chloroethyl)-1-nitrosourea. Cancer Res. 1994; 54:3793-9.

10. Chang JE, Khuntia D, Robins HI, Mehta MP. Radiotherapy and radiosensitizers in the treatment of glioblastoma multiforme. Clin Adv Hematol Oncol. 2007; 5:894-902, 907-15.

11. Van Nifterik KA, van den Berg J, Stalpers LJ. Differential radiosensitizing potential of temozolomide in MGMT promoter methylated glioblastoma multiforme cell lines. Int $\mathrm{J}$ Radiat Oncol Biol Phys. 2007; 69:1246-53.

12. Antonadau D, Paraskevaidis $M$, Sarris $M$. Phase II randomised trial of temozolomide and concurrent radiotherapy in patients with brain metastases. J Clin Oncol 2002; 20(17):3644-50.

13. Verger E, Gil M, Yaya R. Temozolomide and concurrent whole brain radiotherapy in patients with brain metastases: a phase II randomized trial. Int J Radiat Oncol Biol Phys 2005; 61(1):185-91.

14. Wedge SR, Porteous JK, Glaser MG, Marcus $K$, Newlands ES. In vitro evaluation of temozolomide combined with X-irradiation. Anticancer Drugs 1997; 8:92-7.
15. World Health Organization. WHO Handbook for reporting results of cancer treatment. Geneva Offset publication No. 48, 1979; 1-42.

16. Borgelt B, Gelber R, Kramer S, Brady LW, Chang $\mathrm{CH}$, Davis LW, et al. The palliation of brain metastases: Final results of the first two studies by the Radiation Therapy Oncology Group. Int $\mathrm{J}$ Radiat Oncol Biol Phys 1980; 6:1-9.

17. Katz HR. The relative effectiveness of radiation therapy, corticosteroids and surgery in the management of melanoma metastatic to the central nervous system. Int $\mathrm{J}$ Radiat Oncol Biol Phys 1981; 7:897-906.

18. Sneed PK, Larson DA, Wara WM. Radiotherapy for cerebral metastases. Neurosurg Clin N Am 1996; 7:505-15.

19. Posner J. Management of central nervous system metastasis. Sem Oncol 1977; 4:81-91.

20. Tosoni A, Ermani M, Brandes AA. The pathogenesis and treatment of brain metastases: a comprehensive review. Critical Rev Oncol Hematol 2004; 52:199-215.

21. Gamboa-Vignolle C, Ferrari-Carballo T, Arrieta O, Mohar A. Whole-brain irradiation with concomitant daily fixed-dose temozolomide for brain metastases treatment: A randomised phase II trial. Radiother Oncol 2012; 102:187-91.

22. Cross NE, Glantz MJ. Neurologic complications of radiation therapy. Neurol Clin 2003; 21:249-77.

23. Evans ML, Graham MM, Mahler PA, Rasey JS. Use of steroids to suppress vascular response to radiation. Int $\mathrm{J}$ Radiat Oncol Biol Phys 1987; 13:563-7. 